Präv Gesundheitsf 2010 · 5:62-63

DOI 10.1007/s11553-010-0238-1

Online publiziert: 28. April 2010

(c) Springer-Verlag 2010

\author{
A. Gerhardus \\ AG Epidemiologie \& International Public Health, \\ Fakultät für Gesundheitswissenschaften, Universität Bielefeld
}

\title{
Gesundheitsförderung und Evidenz - Gegensatz oder Beziehung mit Potential?
}

Um den Bedarf an individueller Gesundheitsförderung geht es hingegen in dem Beitrag von K. Kummer et al. Sie haben ein Assessmentinstrument zur Erfassung von Ressourcen und Risiken bei älteren pflegenden Angehörigen entwickelt. Aus den Ergebnissen lassen sich maßgeschneiderte gesundheitsförderliche Angebote ableiten.

A. Pieter et al. beschäftigen sich damit, dass gesundheitsförderliche Angebote oft unzureichend angenommen werden. Sie empfehlen „situierte Lernansätze“, die in Abgrenzung zur traditionellen Wissensaufnahme auf interaktive, kontextbezogene und reflektive Elemente setzen. Einen anderen Weg der Wissensaufnahme schlagen A. Zeyer und F. Odermatt vor: Um die Gesundheitskompetenz („health literacy“) zu verbessern, empfehlen sie, Gesundheitsbildung und naturwissenschaftlichen Unterricht zu verknüpfen. Davon profitierten beide Themenfelder: Der naturwissenschaftliche Unterricht gewinnt durch die Integration von Gesundheitsthemen an Attraktivität und vermittelt umgekehrt essentielle Grundlagen für die Gesundheitskompetenz.

Gut belegt ist der Zusammenhang zwischen sozialer Benachteiligung und einem schlechteren Gesundheitsstatus. Unglücklicherweise wird dieser Gradient durch die geringe Akzeptanz von gesundheitsfördernden und präventiven Angeboten durch sozial benachteiligte Gruppen weiter verschärft. T. Brand und T. Jungmann untersuchen, wie mit Hilfe von Multiplikatoren gegengesteuert werden kann. W. Maier und A. Mielck finden in einem systematischen Review eine erhöhte Be- lastung durch Luftverschmutzung und Lärm in den unteren sozialen Statusgruppen. Selbst im Binnenraum eines sozial benachteiligten Wohngebiets verzeichnen D. Koller et al. deutliche Unterschiede im Hinblick auf gesundheitsrelevantes Verhalten und der Zufriedenheit mit der Wohnsituation in Abhängigkeit vom Sozialstatus.

Die Evidenzlage für die gesundheitsfördernden Effekte von sportlicher Bewegung ist in den letzten Jahren zunehmend besser geworden. T. Draxler und $\mathrm{H}$. Ostermann finden in einer Querschnittsuntersuchung bei Personen, die eine asiatische Kampfsportart ausüben, bessere Lebensqualitätswerte als in einer Vergleichspopulation. $\mathrm{Zu}$ Recht verweisen sie auf den explorativen Charakter ihrer Studie, deren Ergebnisse durch Längsschnittuntersuchungen bestätigt werden müssten. Nicht zu den gesundheitsfördernden Sportarten gehört das Glücksspiel in Kasinos - im Gegenteil: Um Menschen mit gesundheitsgefährdender Spielsucht rechtzeitig erkennen zu können, wurde in der Schweiz ein differenzierter Kriterienkata$\log$ entwickelt. In einer Analyse der Daten von auffällig gewordenen Spielern weisen J. Häfeli und S. Lischer jedoch die limitierte Effektivität dieses Instruments nach und zeigen damit eine relevante Evidenzlücke auf.

Eindeutig ist die Evidenzlage, wenn es um die Gefahren des Rauchens geht. A. Rasch et al. motivierte dies dazu, die Krankenkassen in Deutschland zu Angeboten der Raucherentwöhnung zu befragen. Von den antwortenden Kassen bieten fast alle verhaltensbezogene Kurse zur 
Raucherentwöhnung an, obwohl die Evidenz zur Effektivität und Effizienz der Maßnahmen relativ dünn sei. Besser belegt ist der Nutzen für die ebenfalls von den Krankenkassen angebotenen Disease-Management-Programmen (DMP) zur Versorgung des Diabetes mellitus. In einer Längsschnittanalyse von Verordnungsdaten konnten J. Wang et al. eine verbesserte Arzneimittelversorgung im Rahmen der DMP zeigen.

Die Beiträge in dieser Ausgabe decken ein breites Themenspektrum ab. Jeder der Artikel trägt auf seine Art zu einer besseren Evidenzlage in Gesundheitsförderung und Prävention bei - und sei es durch das Aufdecken von relevanten Evidenzlücken.

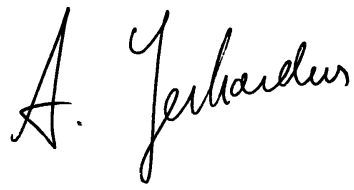

A. Gerhardus

\section{Korrespondenzadresse}

\section{Dr. A. Gerhardus}

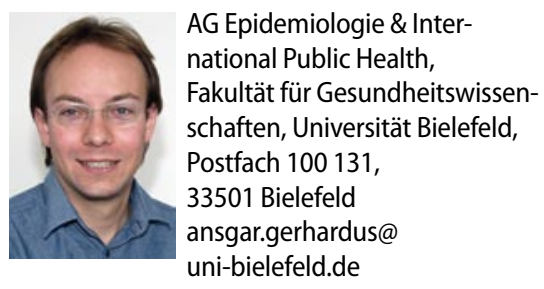

Ansgar Gerhardus, Jürgen Breckenkamp, Oliver Razum, Norbert Schmacke, Helmut Wenzel (Hrsg.)

\section{Evidence-based Public Health}

Bern: Huber 2010, 1. Auflage, 275 S., (ISBN

978-3456847641), broschürt, 29.00 EUR

Schon seit vielen Jahren findet das Prinzip der Evidenz-basierten Medizin (EbM) große Beachtung und Akzeptanz. Ihm liegt zu Grunde, dass bei der Wahl der Diagnostik und Therapie neben der klinischen Expertise des behandelnden Arztes und den Präferenzen des Patienten auch die best verfügbare wissenschaftliche d. h. externe Evidenz einbezogen werden sollte. Um aufgrund der Flut an Veröffentlichungen dem Praktiker die Einbindung der aktuellen Literatur zu erleichtern, wurde zur Wirksamkeit von verschiedenen medizinischen Interventionen die vorhandene Literatur in systematischen Übersichtsarbeiten zusammengefasst. EbM unterstützt den behandelnden Arzt also auf der Ebene der individuellen Therapieentscheidung. Bei Public-Health geht es dagegen darum, bei der Bevölkerung oder ganzen Bevölkerungsgruppen die Gesundheit mit geeigneten Maßnahmen zu erhalten bzw. zu fördern. Public Health fokussiert also nicht auf das einzelne Individuum, sondern hat ganze Gruppen im Visier und zielt darauf ab, Krankheit zu verhüten und rechtzeitig zu erkennen. Wenn auch das Prinzip an sich allgemein anerkannt und eindeutig ist, besteht über die geeigneten Maßnahmen in manchen Situationen Uneinigkeit. Hier stellt sich die Frage, ob die Denkweise der evidenzbasierten Medizin auch im Bereich Public Health Anwendung finden kann.

Auf diese Frage möchten die Autoren mit diesem Buch eine Antwort geben. Nach einem einleitenden Kapitel, in dem der Hintergrund zu EbM kurz und anschaulich beleuchtet wird, wird der Leser anschließend auf eine Studienreise mitgenommen, auf eine Reise durch Evidence-based Public Health (EbMPH). Diese beginnt mit der Vorstellung eines Modells, welches die einzelnen Schritte beinhaltet, die bei der Entwicklung einer geeigneten Maßnahme für ein Public-Health-Problem unter hinzuziehen von EbM zu durchlaufen sind. Im Folgenden werden die einzelnen Schritte dann in separaten Kapiteln ausführlich erläutert. Der Leser findet Unterstützung durch viele anschauliche Beispiele, die es erleichtern, das Prinzip zu verstehen. Die Reise beginnt mit der Erkennung des Gesundheitsproblems, geht über die Bestimmung der dafür relevanten Fragestellungen hin zum Kern von EbMPH, zur Auswahl der Methoden und der Erstellung der Evidenz. Dort finden neben gesundheitlichen Aspekten, auch ethische, ökonomische, rechtliche und soziokulturelle Aspekte Beachtung. Es schließt sich ein ganz praktischer und überaus wichtiger Teil an: Tipps, wie man die neu gewonnenen Erkenntnisse später anderen gegenüber kommunizieren kann. Im letzten und fünften Teil kann der Leser sein gewonnenes Wissen anhand weiterer Beispiele aus dem Bereich der Gesundheitswissenschaften testen und festigen (z. B. Influenza-Pandemie, Screening Zervixkarzinom, chronischer Rückenschmerz). Das Buch endet mit einem Fazit und einem kurzen Ausblick.

Die Autoren zeigen mit diesem Buch, dass es - auch wenn das Anliegen von Public Health klar ist - durchaus wissenschaftlich fundiert zu gucken gilt, mit welchen Mitteln die Umsetzung der Ziele geschehen soll. Es ist somit nicht nur für Studenten sondern auch für im Gesundheitswesen tätigen Personen und Wissenschaftler eine empfehlenswerte Lektüre. Die Idee der Studienreise gibt dem Buch eine klare Struktur und lädt ein, sich mit dem Thema näher zu beschäftigen. Die Reise hält viele neue Eindrücke bereit, stellt gewohnte Perspektiven in Frage und gibt dem Leser viele neue Denkanstöße. Die Sprache ist sehr gut verständlich, die vielen aktuellen Beispiele sind überaus hilfreich und die Tipps eine besondere Ergänzung. Bei Interesse kann der erste Einblick in die allgemeine EbM des ersten Kapitels sicherlich noch mit weiterführender Literatur vertieft werden. Die anfangs gestellte Frage kann nach der Lektüre nur mit ,ja“ beantwortet werden. Ein jeder sollte dies jedoch für sich selber mit Hilfe dieses überaus lesenswerten Buches herausfinden.

Ursula Schütte (Dresden) 